\title{
Óleos essenciais de espécies do gênero Aniba
}

A. ALPANDE DE MORAIS(3), C. M. ANDRADE DA MATA REZENDE(3), 'M. V. VON BULOW(3), J. CORREA MOURÃO(3), O. R. GOTTLIEB(3), M. C. MARX(3), A. I. DA ROCHA(1) e M. TAVEIRA MAGALHÁES(2).

\section{SINOPSE}

A maior parte das espécies de Aniba (Lauraceae) se encontra na Amazônia. São representadas por árvores que contêm óleo essencial em todos os set's orgãos. Os óleos dos diferentes orgãos de qualquer espécie mostram somente uma pequena variação em sua composição. Em contrapartida, pode ser notada uma variação tão surpreendentemente drástica entre espécies diferentes que podem ser classificadas em grupos. O grupo linalol compreende A. duckei Kos term. e A. rosaeodora Ducke. O grupo benzoato de benzilo compreende A. burchellii Kosterm., A. firmula (Nees \& Mart.) Mez., A. fragrans Ducke, A. gardneri (Meissn.) Mez, A. guianensis Aubl., A. parvifiora (Meissn.) Mez. e A. permollis (Nees) Mez. O grupo alilbenzeno compreende A. canelilla (H.B.K.) Mez., A. hostmanniana (Nees) Mez e A. pseudocoto (Rus by) Kosterm. A análise detalhada revelou a presen. ça, em alguns destes óleos ,de produtos raros de plan. tas como 1-nitro-2feniletano, benzoato de feniletilo O-metilisoeugenol e 2, 4, 5-trimetoxialilbenzeno.

Duas espécies vegetais têm sido exploradas para a obtenção do óleo essencial de paurosa. A primeira, oriunda da Guiana Francesa, tornou-se praticamente extinta por volta de 1932. A segunda foi encontrada em 1927 na Amazônia brasileira, constituindo-se na espécie que, até hoje, serve de fonte para o óleo comercializado (Guenther, 1950). As classificações botânicas aessas espécies foram feitas por Ducke, que denominou a espécie da Guiana Francesa de Aniba rosaeodora (Ducke, 1930), e por Kostermans, que denominou a espécie amazônica de Aniba duckei (Kostermans, 1958). 0 componente principal do óleo de ambas as espécies é o linalol, constituindo cerca de $80 \%$ no óleo brasileiro e mais de $90 \%$ no óleo da Guiana (Guenther, 1950). Quando o óleo brasileiro é obtido de plantas jovens apresenta teores de linalol maiores que $90 \%$ (Gottlieb et alî, 1964).
Como resultado da derrubada sucessiva das árvores silvestres, Aniba duckei está se tornando cada vez mais escassa. Numa tentativa de evitar que se perca esta fonte de renda da Amazônia, tal como foi perdida pela Guiana Francesa, decidimos investigar o problema por diferentes caminhos. Um deles foi o de estudar os óleos essenciais de espécies do gênero Aniba, na expectativa de encontrar alguma capaz de substituir a Aniba duckei como produ. tora do óleo de pau-rosa.

Após o exame de doze espécies de Aniba, número significativo, tendo em conta que se julga existirem aproximadamente quarenta (Kostermans, 1957), podemos distribuir essas espécies em três grupos, de acordo com a natureza química do constituinte predominante do respectivo óleo essencial.

\section{GRUPO DO LINALOL}

A este grupo, pelo menos por ora, pertencem apenas Aniba rosaedora e Aniba duckei Guenther, 1950; Gottieb et alii, 1964).

\section{GRUPO DO BENZOATO}

A este grupo pertencem as espécies em que o benzoato de benzila é o componente principal. Em virtude dos óleos terem sido estudados em épocas bastante distintas, observa-se que não houve uniformidade nas técnicas utilizadas para a identificação dos respectivos componentes (Tabela 1),

O óleo de Aniba fragrans foi submetido a uma destilação fracionada. A fração leve foi identificada com o linalol pelo ponto de ebulição; a fração pesada foi identificada com o benzoato de benzila por espectrometria no infravermelho e por hidrólise, seguida da identificação do álcool benzílico e do ácido benzoico.

(1) - Instituto Nacional de Pesquisas da Amazônia, Conselho Nacional de Pesquisas, Manaus.

(2) - Instituto de Tecnologia Alimentar, Ministério da Agrícultura, Rio de Janeiro.

(3) - Universidade Federal Rural do Rio de Janeiro. 
O óleo de Aniba firmula, tratado com uma solução de hidróxido de sódio, produziu um precipitado que foi separado por filtração. A acidificação deste precipitado regenerou uma substância que foi identificada com o salicilato de benzila pelos espectros no infravermelho e de ressonância magnética nuclear. Destilação fracionada do óleo residual forneceu uma fra- ção leve de monoterpenos, caracterizados por cromatografia gás-líquido, e uma fração pesada constituída por benzoato de benzila (componente predominante) e por benzoato de $\beta$-feniletila, ambos caracterizados pelas espectrometrias no infravermelho e de ressonância magnética nuclear.

TABELA 1

COMPOSIÇÃO PERCENTUAL DOS ÓLEOS ESSENCIAIS DE ESPÉCIES DE ANIBA:

GRUPO DO BENZOATO

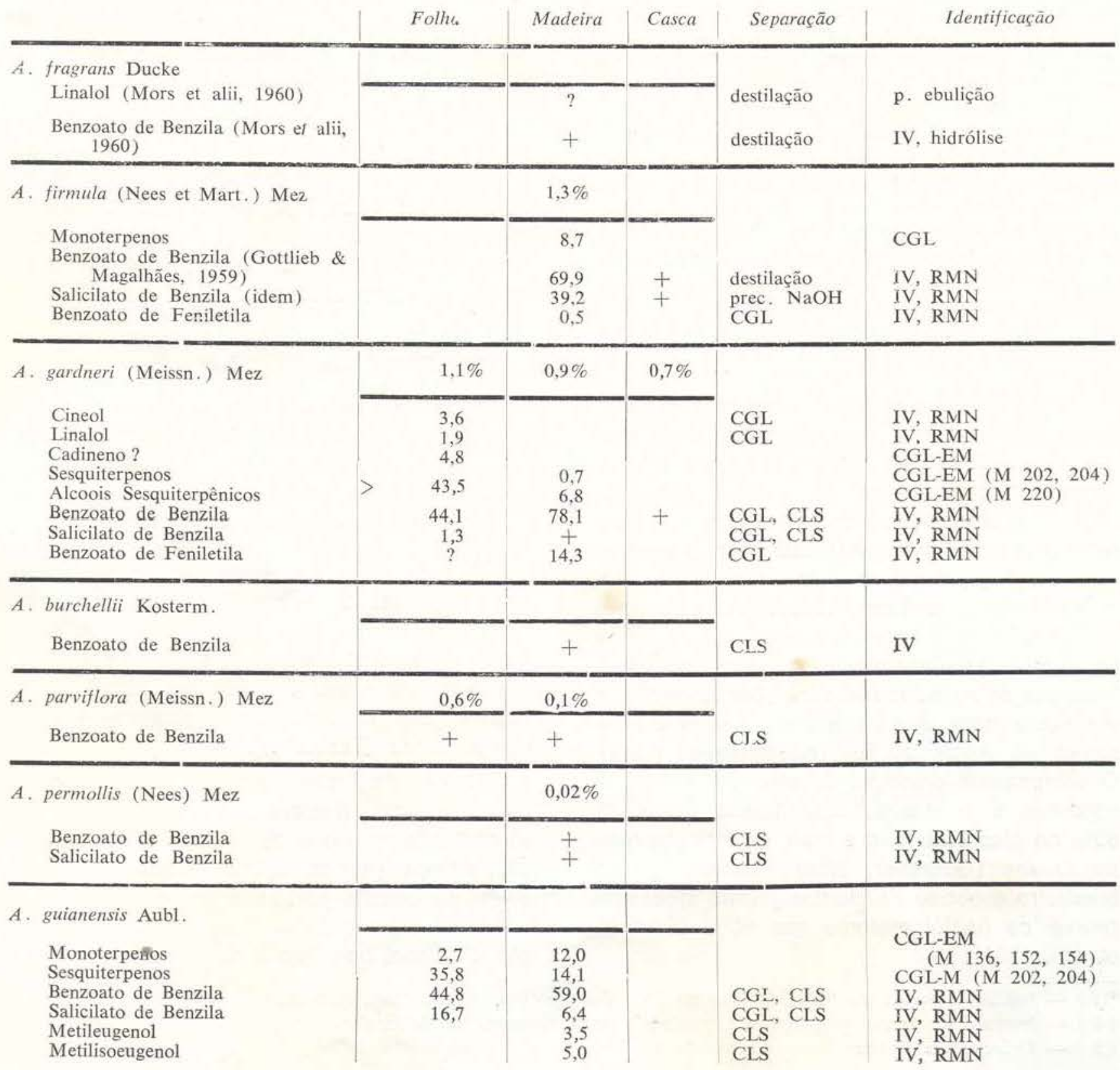


Os óleos das folhas e dos galhos da Aniba gardneri foram estudados por cromatografia gás-líquido, analítica e preparativa. Os constituintes destes óleos foram isolados e subme. tidos às espectrometrias no infravermelho, de resconância magnética nuclear e de massa. O 1,8-cineol e o linalol foram identificados por comparação dos espectros no infravermelho e de ressonância magnética, com espectros de padiões. O cadideno foi identificado, tentativamente, por espectrometria de massa. Sesquiterpenos e álcoois sesquiterpênicos tiveram sua natureza determinada por espectrometria de massa. Benzoato e o salicilato de benzila. assim como benzoato de $\beta$-feniletila foram identificados por cromatografia gás-líquido, espectrometria no infravermelho, espectrometria de ressonância magnética nuclear e espectrome tria de massa. Observa-se uma distribuição equilibrada de terpenos e de ésteres aromáticos no óleo das folhas, enquanto que no óleo da madeira nota-se acentuada predominância de ésteres aromáticos.

Nos óleos de Aniba burchellii e de Aniba parviflora identificou-se, por ora, apenas o benzoato de benzila.
No óleo de Aniba permollis, identificaramse benzoato e salicilato de benzila, tendo sido eles separados por cromatografia líquido-sólido.

Nos óleos das folhas e dos galhos da Aniba guianensis, pelaś técnicas já referidas, identificaram-se monoterpenos, sesquiterpenos e esteres aromáticas, estes em maior percentagem. Embora em quantidades relativamente pequenas, ccorrem ainda compostos alilbenzênicos

\section{GRUPO DO ALILBENZENO}

A este grupo pertencem três espécies até agora estudadas: Aniba canelilla, Aniba hostmanniana e Aniba pseudocoto (Tabela 2) .

Os óleos essenciais da casca, do alburno e do cerne da Aniba canelilla contêm principalmente $\beta$-nitrofeniletano e metileugenol, ocorrendo ainda pequenas quantidades de terpenos $\rightarrow$ de eugenol. Estes constituintes foram isolados por cromatografia gás-líquido e cromatografia líquido-sólido e identificados por métodos ospectrométricos e métodos químicos.

Noe óleos obtidos da madeira e da casca de Aniba hostmaniana encontra-se, principalmente, 2, 4, 5-trimetoxialilben $<$ eno, ocorrendo ainda pequenas quantidades de monoterpenos e de metileugenol.

TABELA 2

COMPOSIÇÃO PERCENTUAL DOS ÓLEOS ESSENCIAIS DE ESPÉCIES DE ANIBA: GRUPO DO ALILBENZENO

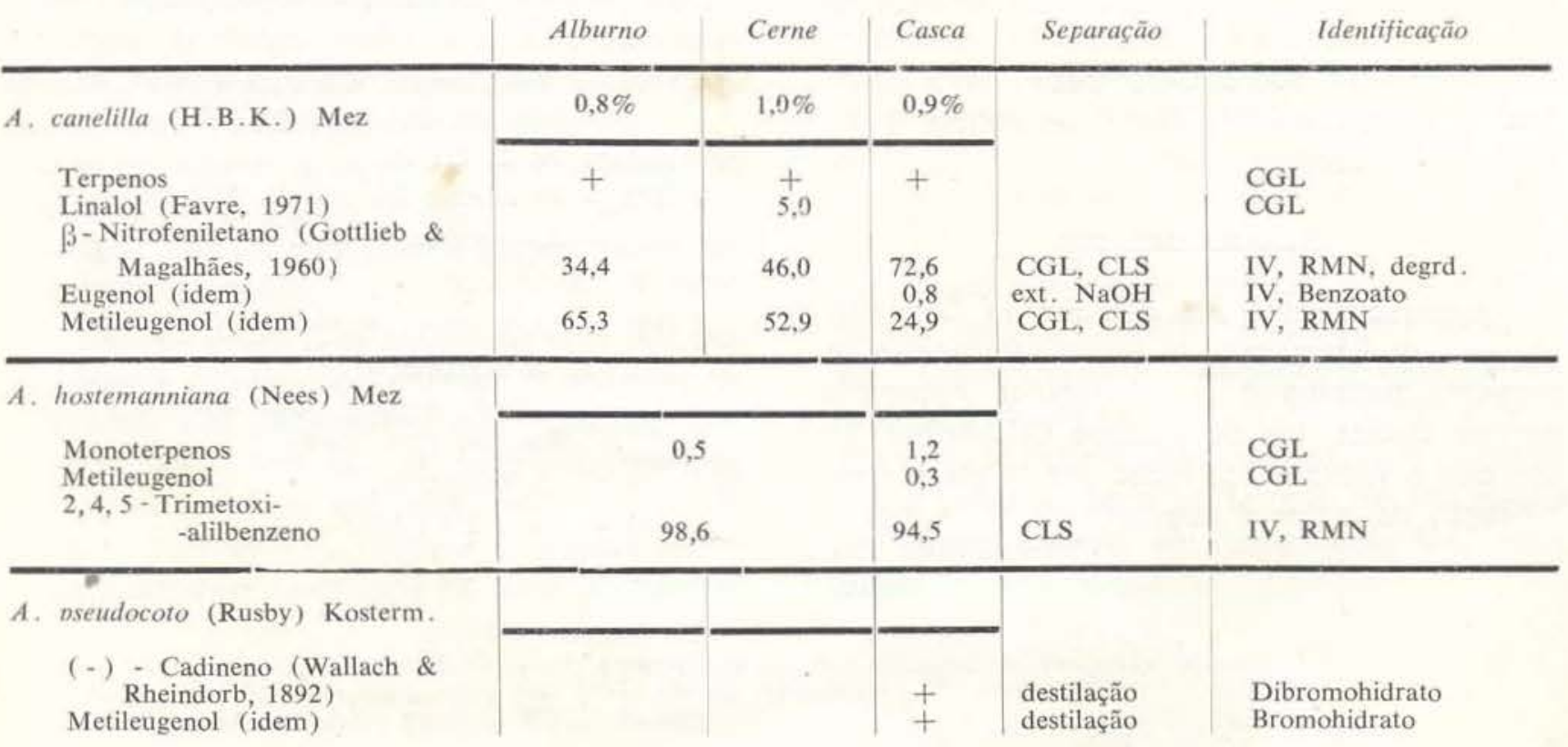


O óleo essencial da casca da Aniba pseu. docoto, espécie boliviana originalmente descrita como Ocotea pseudocoto, contêm principalmente cadineno e metileugenol. Estas substâncias foram separadas por destilação fracionada e identificadas através da preparação de derivados por Wallach e Rheindorf (1892).

\section{Comentários}

Após o exame de doze espécies de Aniba, tornou-se claro que é pequena a probabilidade de encontrar uma espécie do gênero, capaz de substituir a Aniba duckei como produtora do óleo essencial de pau-rosa. Ainda que não tẹnha sido possivel solucionar o problema indus trial em mira, os estudos realizados foram bastante interessantes do ponto de vista científico. Benzoato e salicilato de benzila são substâncias comumente encontradas em flores ( $\mathrm{Na}$ ves \& Mazuyer, 1947). Sua ocorrência em madeiras sãs, entretanto, é apontada em raras insiâncias. Benzoato de - feniletila, - nitrofeniletano, 2, 4, 5-trimetoxi-akilbenzeno e metiliso-eugenol são frodutos raramente encontrados em óleos essenciais.

\section{EXPERIMENTAL}

CGL analítica de óleos do grupo do benzoato foi realizada em colunas de $2 \mathrm{~m} \times 3 / 16$ " com $20 \%$ de Carbowax 20M sobre Chromosorb P a $220^{\circ}$. Temp. do injetor $260^{\circ}$. Temp. do detetor $280^{\circ}$. Vazão do hidrogênio $80 \mathrm{ml} / \mathrm{min}$. CGL analítica de óleos do grupo alilbenzeno foi reaiiazada em colunas de $2 \mathrm{~m} \times 1 / 4$ " com $20 \%$ de Apiezon M sobre Chromosorb W a $200^{\circ}$. Temp. do injetor $240^{\circ}$. Temp. do detetor $280^{\circ}$. Vazão do hidrogênio $80 \mathrm{ml} / \mathrm{min}$.

\section{AGRAdeCIMENTOS}

Agradecimentos são devidos ao Conselho Nacional de Pesquisas por suporte financeiro ao presente trabalho e aos botânicos Apparício Pereira Duarte, Ida de Vattimo Gil, Arthur A. Loureiro e William Rodrigues por coleta e clas. sificação do material vegetal.

\section{SUMMARY}

Most Aniba (Lauraceae) species occur in Ama zonia. They are represented by trees which contain essential oil in all of their organs. Oils from diffe. rent organs of any species show little variation in composition. In contradistinction, such surprisingly drastic variation may be noted among different spe cies, that they can be classified into groups. The linalool - group comprises A. duckei Kosterm. and A. rosaeodora Ducke. The benzyl benzoate - group comprises A. burchellii Kosterm., A. firmula (Nees et Nart.) Mez., A. fragrans Ducke, A. gardneri (Meissn.) Mez, A. guianensis Aubl., A. parviflora (Meissn.) Mez and A. permollis (Nees) Mez. The allylbenzene group comprises A. canelilla (H.B.K.) Mez, A. host manniana (Nees) Mez and A. pseudocoto (Rusby) Kosterm. Detailed analysis revealed the presence in some of these oils of such rare plant products as 1-nitro-2-phenylethane, pheny'athyl benzoate, O-me thylisoeugenol and 2, 4, 5.trimethoxyallylbenzene.

\section{BIBLIOGRAFIA CITADA}

DUCKE, A .

1930 - Arch. Jard. Bot. Rio de Janeiro, 5: 101.

FAVRE, $R$.

1971 - In V Congresso Internacional de Oleos essen ciais, São Paulo.

GotTLIEB, O. R. ET ALII

1964 - Perf. Essent. Oil Record, 55: 253.

Gottlleb, O. R. \& Magalhães, M. Taveira

1959a - J. Org. Chem., 24: 2070

1959b - Perf. Essent. Oil Record, 50: 119.

1960 - Perf. Essent. Oil Record, 51: 69.

GUENTHER, E

1950 - "The Essential Oils". New York, D. van Nostrand co. v. 4, p. 183.

Kostermans, A. J. G. H.

1938 - Rec. trav. bot. neerl., 35: 866 .

1957 - Reinwardtia, $4: 2,193$.

MORS, W. B. ET ALII

1960 -- Anais Assoc, brasil. Quim., 19: 193.

Naves, Y. R. \& MAzUyer, G.

1947 - Natural perfume Materia's. New York. Reinhold Publ. corp.

WALLACH, O \& RHEINDORF, T.

1892 - Ann., 271: 300 